This is an electronic reprint of the original article. This reprint may differ from the original in pagination and typographic detail.

\author{
Author(s): Shalashov, A. G.; Gospodchikov, E. D.; Izotov, I. V.; Mansfeld, D. A.; Skalyga, V. A.; \\ Tarvainen, Olli
}

Title: $\quad$ Observation of Poincaré-Andronov-Hopf Bifurcation in Cyclotron Maser Emission from a Magnetic Plasma Trap

Year: $\quad 2018$

Version:

Please cite the original version:

Shalashov, A. G., Gospodchikov, E. D., Izotov, I. V., Mansfeld, D. A., Skalyga, V. A., \& Tarvainen, O. (2018). Observation of Poincaré-Andronov-Hopf Bifurcation in Cyclotron Maser Emission from a Magnetic Plasma Trap. Physical Review Letters, 120(15), Article 155001. https://doi.org/10.1103/PhysRevLett.120.155001

All material supplied via JYX is protected by copyright and other intellectual property rights, and duplication or sale of all or part of any of the repository collections is not permitted, except that material may be duplicated by you for your research use or educational purposes in electronic or print form. You must obtain permission for any other use. Electronic or print copies may not be offered, whether for sale or otherwise to anyone who is not an authorised user. 


\title{
Observation of Poincaré-Andronov-Hopf Bifurcation in Cyclotron Maser Emission from a Magnetic Plasma Trap
}

\author{
A. G. Shalashov, ${ }^{1, *}$ E. D. Gospodchikov, ${ }^{1}$ I. V. Izotov, ${ }^{1}$ D. A. Mansfeld, ${ }^{1}$ V. A. Skalyga, ${ }^{1}$ and O. Tarvainen ${ }^{2}$ \\ ${ }^{1}$ Institute of Applied Physics, Russian Academy of Sciences (IAP RAS), 46 Ulyanova st., 603950 Nizhny Novgorod, Russia \\ ${ }^{2}$ Department of Physics, University of Jyvaskyla, PO Box 35 (YFL), 40500 Jyvaskyla, Finland
}

(Received 15 December 2017; revised manuscript received 21 February 2018; published 11 April 2018)

\begin{abstract}
We report the first experimental evidence of a controlled transition from the generation of periodic bursts of electromagnetic radiation into the continuous-wave regime of a cyclotron maser formed in magnetically confined nonequilibrium plasma. The kinetic cyclotron instability of the extraordinary wave of weakly inhomogeneous magnetized plasma is driven by the anisotropic electron population resulting from electron cyclotron plasma heating in a MHD-stable minimum- $B$ open magnetic trap.
\end{abstract}

DOI: 10.1103/PhysRevLett.120.155001

Electron cyclotron instabilities caused by resonant interaction between energetic electrons and electromagnetic waves are typical for open plasma traps with magnetic mirrors. Studies of the cyclotron instabilities of nonequilibrium plasmas have led to the plasma cyclotron maser paradigm, which explains a rich class of phenomena of coherent radioemission from Earth's magnetosphere [1-3], the Solar corona [4-10], other astrophysical objects [11-14], and laboratory magnetic traps [15-28]. Unlike vacuum electronic devices based on the electron cyclotron resonance (ECR) [29], stimulated emission from plasma traps is usually very far from being monochromatic. The obvious reason is the lack of electromagnetic mode selection by an external cavity typical for masers and lasers. A more sophisticated reason is that the kinetic instabilities are driven by regions with free energy in the phase space of resonant particles, most commonly by the inverse population over Landau levels; i.e., $\partial f / \partial v_{\perp}>0$. In natural conditions, both space and laboratory, the distribution function of fast electrons is widely spread over the momentum space and inhomogeneous in the real space. The development of the cyclotron instability under such conditions results in the generation of periodic, quasiperiodic, or stochastic broadband pulses of emission. Each electromagnetic pulse is accompanied by pulsed precipitations of fast electrons from the trap as the electrons lose their transverse momentum and fall into the loss cone due to the interaction with waves. Owing to the sharp decrease in the free energy, the system falls under the instability threshold; after that, a preparation of the subsequent burst (accumulation of resonant particles) begins, and the process repeats [1]. Indeed, starting from early works, the existence of oscillatory regimes for constant strength of a particle source has been understood as a general property of plasma systems with quasilinear relaxation [30-33].

A continuous-wave $(\mathrm{cw})$ generation is also possible when the system stays near the instability threshold and the accumulation and emission phases are not separable; i.e., the number of high-energy particles delivered by a source is constantly equal to the number of precipitating particles. The steady-state emission of plasma cyclotron maser was extensively studied theoretically (see Ref. [1] and references therein), but it has never been detected reliably in a laboratory because of the narrow region of plasma parameters where the regime exists.

In this Letter, we present the first experimental evidence of the controlled transition between the burst and $\mathrm{cw}$ regimes of the electron cyclotron instability developing in the microwave range in an open magnetic trap and discuss the related physics. This transition is related to the Poincaré-Andronov-Hopf bifurcation; namely, a stationary point attributed to $\mathrm{cw}$ generation becomes unstable through the birth of a stable limit cycle. A similar transition is known for optical lasers with nonlinear filters [34]. In contrast to lasers, in which the active matter and the nonlinear absorber can be tuned independently, in the plasma maser both the gain and dissipation are governed by the same nonequilibrium electron distribution function. In this case, the transition to the $\mathrm{cw}$ regime requires fine tuning of the source of nonequilibrium electrons in the phase space; in our experiment, we found a way to implement such adjustment.

Let us consider first the theoretical model that motivates the experiment. A self-consistent evolution of particles and waves may be described by the quasilinear theory, a perturbative approach that involves many overlapped waveparticle resonances as a basis for diffusive particle transport in the phase space $[35,36]$. When the cyclotron instability evolves slowly in comparison to the bounce oscillations of resonant electrons in an open trap and a narrow frequency spectrum of wave turbulence is assumed, the following set of bounce-averaged quasilinear equations can be formulated as $[1,33]$ 


$$
\begin{aligned}
& \frac{\partial F}{\partial t}=E \frac{\partial}{\partial \kappa}\left(D \frac{\partial F}{\partial \kappa}\right)+J \\
& \frac{\partial E}{\partial t}=E \int_{0}^{\infty} \int_{\kappa_{c}}^{1} K \frac{\partial F}{\partial \kappa} d \kappa d v-\nu E,
\end{aligned}
$$

where $F(t, \kappa, v)$ is the electron distribution over the invariants of adiabatic motion $\kappa=\left(v_{\perp} / v\right) \times\left(B_{\min } / B\right)^{1 / 2}$, $v$ and $v_{\perp}$ are the absolute velocity and its component perpendicular to the external magnetic field $B, \kappa_{c}=$ $\left(B_{\min } / B_{\max }\right)^{1 / 2}$ is the loss-cone boundary, $D(\kappa, v)$ and $K(\kappa, v)$ are known smooth positive functions [1], and $J(\kappa, v)$ is a stationary source of nonequilibrium electrons. The diffusion takes into account the electron scattering by unstable waves. The larger the average wave energy $E(t)$ in a flux tube, the faster is the electron diffusion into the loss cone, which is the dominant mechanism of the electron loss. The wave energy, in turn, is determined from the averaged transport equation, in which the instability growth rate is proportional to $\partial F / \partial \kappa$, and $\nu$ stands for the wave dissipation rate due to damping in the background plasma and convective losses.

For the case adequate to our experiment - the right-handpolarized extraordinary wave at the fundamental harmonic propagating at a small angle to the magnetic field-one can assume $D \approx \mathcal{D}(\kappa) \propto \kappa$ and $K \approx \chi(v) \mathcal{K}(\kappa) \propto v^{4} \kappa^{2}$. Then, it is sufficient to consider $\mathcal{F}(t, \kappa)=\int_{0}^{\infty} F(t, \kappa, v) \chi(v) d v$ instead of $F(t, \kappa, v)$ and the same for $J(\kappa, v) \rightarrow \mathcal{J}(\kappa)$. One can seek a solution of Eqs. (1) as a series over the eigenmodes $\phi_{n}$ of the quasilinear diffusion operator,

$$
\mathcal{F}=\sum_{n=1}^{\infty} f_{n}(t) \phi_{n}(\kappa), \quad \mathcal{J}=\sum_{n=1}^{\infty} j_{n} \phi_{n}(\kappa) .
$$

Then, Eqs. (1) become

$$
\frac{d f_{n}}{d t}=j_{n}-\mu_{n} f_{n} E, \quad \frac{d E}{d t}=\left(\sum_{n=1}^{\infty} k_{n} f_{n}-\nu\right) E .
$$

Here, $\phi_{n}$ and $k_{n}$ are defined via

$$
\frac{\partial}{\partial \kappa} \mathcal{D} \frac{\partial \phi_{n}}{\partial \kappa}=-\mu_{n} \phi_{n}, \quad k_{n}=\int_{\kappa_{c}}^{1} \mathcal{K} \frac{\partial \phi_{n}}{\partial \kappa} d \kappa,
$$

with the proper boundary conditions $\phi_{n}\left(\kappa_{c}\right)=0, \phi_{n}{ }^{\prime}(1)=0$ and the normalization $\int_{\kappa_{c}}^{1} \phi_{n}^{2} d \kappa=1$. Setting all time derivatives to zero, one can find a steady-state solution with nonzero wave energy,

$$
f_{n}^{*}=j_{n} /\left(\mu_{n} E^{*}\right), \quad E^{*}=\nu^{-1} \sum_{n=1}^{\infty} k_{n} j_{n} / \mu_{n} .
$$

To study the stability of this state, let us note that eigenvalues $\mu_{n}$ are rapidly growing with $n$. Therefore, one can simplify the analysis by keeping only the lowest eigenmode $f_{1}$ in Eq. (2) and assuming all other modes adiabatically varying in time, $f_{n}=j_{n} /\left(\mu_{n} E\right)$ for $n>1$ [1]. Seeking perturbation of the resulting second-order equation near the stationary solution (3) as $\delta E, \delta f_{1} \propto \exp (\lambda t)$, one finds the characteristic equation

$$
\lambda^{2}+\left(\mu_{1} E^{*}+\sum_{n=2}^{\infty} \frac{k_{n} j_{n}}{\mu_{n} E^{*}}\right) \lambda+\mu_{1} \nu E^{*}=0 .
$$

The stability boundary corresponds to $\operatorname{Re} \lambda=0$. The steady state is unstable when $\lambda$ is real or, equivalently, the term in the brackets is negative. As seen from Eq. (3), the sum in Eq. (4) is of order $\nu$. For simplicity, we may assume a weak source of nonequilibrium electrons, $\mu_{1} E^{*} \ll \nu$; then the instability criterion is independent of the source power; namely,

$$
\sum_{n=2}^{\infty} k_{n} j_{n} / \mu_{n}<0 .
$$

Numerical integration of the complete set of balance equations (2) shows that the condition (5) predicts well the birth of a stable attractor (a limit cycle) for all conditions relevant for the experiments discussed hereafter.

We find that a key factor controlling the steady-state stability is the angular structure $\left\{j_{n}\right\}$ of the particle source. Let us assume that hot electrons are accelerated by an external wave field with a frequency $\omega$ under off-center ECR conditions in an open magnetic configuration. When the ECR surface is shifted outside the trap center and the plasma is rarefied for the heating wave (the parallel refraction index is less than one, i.e., $\left.k_{\|} c / \omega<1\right)$, the cyclotron interaction modifies the bounce oscillations of a resonant electron along the magnetic field lines so that the turning points move towards the ECR $[37,38]$. In the absence of other interactions, such an electron would eventually turn exactly at the point of hot cyclotron resonance $\omega=\omega_{B} / \gamma$, where $\gamma=\left(1-v^{2} / c^{2}\right)^{-1 / 2}$ accounts for the cyclotron frequency downshift due to relativistic mass dependence; the Doppler shift $k_{\|} v_{\|}$is exact zero. One may assume that all particles produced by the source have the same pitch angle in our notation

$\mathcal{J}=J_{0} \delta\left(\kappa-\kappa_{t}\right), \quad j_{n}=J_{0} \phi_{n}\left(\kappa_{t}\right), \quad \kappa_{t}=\sqrt{B_{\min } / \gamma B_{\mathrm{ECR}}}$,

where $B_{\mathrm{ECR}}$ is the magnetic field strength corresponding to the cold ECR. Thus, one may control the instability condition (5) by varying $B_{\min } / B_{\mathrm{ECR}}$ in a laboratory experiment.

To illustrate this point, let us consider the simplified case when $\mathcal{D}, \mathcal{K}=$ const. Then $\mu_{n}=\frac{1}{4} \pi^{2} a^{2}\left(n-\frac{1}{2}\right)^{2} \mathcal{D}$, $\phi_{n}=\sqrt{a} \sin \left[\sqrt{\mu_{n} / \mathcal{D}}\left(\kappa-\kappa_{c}\right)\right]$, and $k_{n}=(-1)^{n+1} \sqrt{a} \mathcal{K}$ with $a=2\left(1-\kappa_{c}\right)^{-1}$. Since $k_{1} j_{1}>0$, the lowest mode is always destabilizing in Eqs. (2). If all modes with $n>2$ are ignored, Eq. (5) leads to $k_{2} j_{2}<0$. In other words, the 

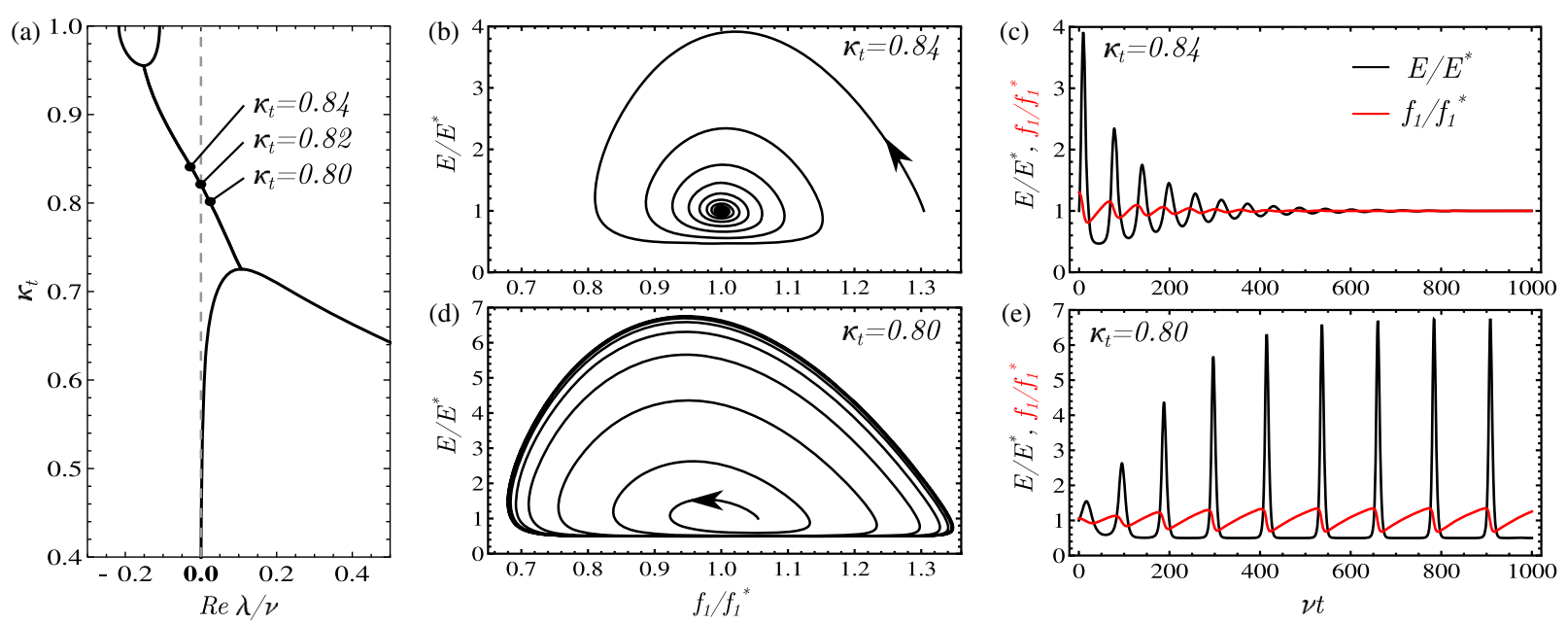

FIG. 1. (a) Solution of the characteristic equation (4), (b),(d) the characteristic phase trajectories, and (c),(e) the time evolution of $E$ and $f_{1}$ from Eqs. (2) in the cw regime (b),(c) and limit cycle regime (d),(e). The wave energy and number of particles in the $\phi_{1}$ mode are normalized over their stationary values (3). The parameters $J_{0} k_{1} / \nu^{2}=0.01$ and $\kappa_{\mathrm{c}}=0.39$ are chosen to fit the experimental data; the first six modes are taken into account in the numerical calculations. The Hopf bifurcation occurs at $\kappa_{t}=0.82$.

stationary generation is stable when both modes are destabilizing, and the limit cycle is stable when the second mode acts as a nonlinear absorber. The boundary between the regimes can be defined from $j_{2}\left(\kappa_{t}\right)=0$, which results in a universal value of the bifurcation magnetic field $B_{\min } / B_{\mathrm{ECR}} \equiv \gamma \kappa_{t}^{2}=\frac{4}{9} \gamma\left(1+\kappa_{c} / 2\right)^{2}$. For the fixed ECR heating frequency, the stationary generation corresponds to a higher magnetic field in comparison to the burst regime.

The same results are obtained for $\mathcal{D} \propto \kappa$ and $\mathcal{K} \propto \kappa^{2}$ that describe our experiment. Here, $\phi_{n}(\kappa)$ are expressed analytically via Bessel functions, while $\mu_{n}$ must be found numerically. Figure 1 shows an example of such calculations for the parameters relevant to our experiment.

The experiment was performed with the ECR ion source (A-ECR-U type) at the JYFL accelerator laboratory [39]; see Fig. 2. The magnetic field was generated by two solenoid coils and a permanent sextupole magnet forming a minimum- $B$ field configuration [40]. A steady-state ECR plasma discharge was supported by microwaves from a traveling wave tube (TWT) amplifier $(250 \mathrm{~W}$ power at $11.8 \mathrm{GHz}$ ). While direct measurements are technically impossible due to a compact design of the setup, the plasma density is estimated as $\sim 5 \times 10^{11} \mathrm{~cm}^{-3}$ based on the experience in similar setups [41]. The electron energy distribution supposedly consists of three components: cold electrons with an average energy of 10-100 eV, warm 1-10 keV electrons, and hot electrons with energies up to $1 \mathrm{MeV}$ [42-44].

The minimum value of the magnetic field $B_{\min }$ was achieved on the axis in between the coils. The ECR condition at the fundamental harmonic was satisfied on a closed nearly ellipsoidal surface with the constant magnetic field $B_{\mathrm{ECR}}=0.42 \mathrm{~T}$. The ECR surface size, characterized with $B_{\min } / B_{\mathrm{ECR}}$, was controlled by varying the coil currents. In the experiments, we run a continuous magnetohydrodynamically stable ECR discharge in oxygen at pressure of $(4-5) \times 10^{-7}$ mbar and tuned the control parameter in the range $B_{\min } / B_{\mathrm{ECR}}=(0.75-0.99) \pm 1.5 \%$. To reach this range, we reduced the heating frequency from the nominal value of $14 \mathrm{GHz}$.

Plasma microwave emission was detected with a highbandwidth digital oscilloscope allowing direct recording of the waveforms of electromagnetic field emitted by the plasma with temporal resolution of $12.5 \mathrm{ps}$. More details on

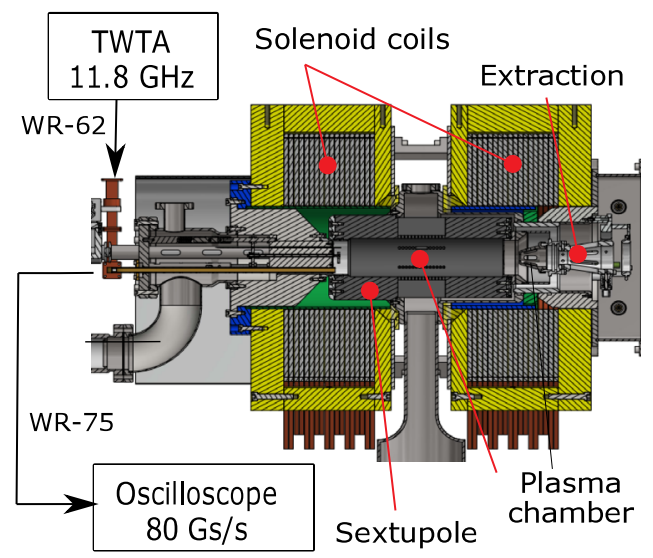

FIG. 2. Schematic of the experimental setup. Microwaves are launched through a WR-62 waveguide port, while the plasma emission is measured through a WR-75 waveguide port. The transmission line to the oscilloscope (Keysight DSOV334A, $80 \mathrm{Gs} / \mathrm{s}$ sampling rate and $33 \mathrm{GHz}$ bandwidth) includes high voltage break, waveguide-to-coaxial transition, power limiter, and tunable attenuator; the frequency response of the line is constant in the range of $8-15 \mathrm{GHz}$. The mirror ratios are $B_{\max } / B_{\min }=4.7-6.8$ and 2.4-3, correspondingly, in the injection (left) and extraction (right) mirrors. 

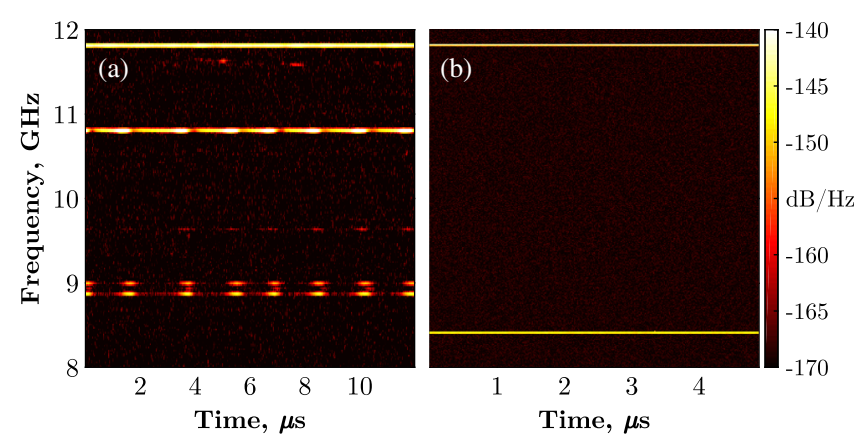

FIG. 3. Dynamic spectra of the plasma emission in the 8$12 \mathrm{GHz}$ range in the (a) pulsed regime at $B_{\min } / B_{\mathrm{ECR}}=0.935$, and the (b) cw regime at $B_{\min } / B_{\mathrm{ECR}}=0.947$. The spectrograms were calculated off-line by short-time Fourier transform with a Hamming window. The horizontal line at $11.8 \mathrm{GHz}$ corresponds to the stray radiation at the TWT amplifier frequency.

the diagnostic technique can be found in Refs. [45,46]. An example of dynamic spectrograms in the frequency band of $8-12 \mathrm{GHz}$ is presented in Fig. 3. The emission spectrum consists of several narrow-band discrete lines with the linewidth less than $30 \mathrm{MHz}$.

The experimental findings can be summarized as follows. No enhanced cyclotron (maser) emission from the plasma is observed in low magnetic fields when $B_{\min } / B_{\mathrm{ECR}}<0.88$. A reproducible generation of quasiperiodic bursts near the fundamental and second electron cyclotron harmonics is observed at $B_{\min } / B_{\mathrm{ECR}}=0.88-0.93$. The cw generation near the fundamental harmonic is detected at stronger field when $B_{\min } / B_{\mathrm{ECR}}=0.94-0.98$. With further increase of the magnetic field, plasma heating becomes inefficient since the ECR absorption volume is small, and the cyclotron instability shows stochastic features.

Typical emission patterns in the burst and cw regimes are presented in Fig. 4. Plot (a) is related to the burst regime of the electron cyclotron instability. The microwave signal consists of a series of wave packets with duration of $1 \mu \mathrm{s}$ and repetition period of $2 \mu \mathrm{s}$. Depending on the experimental conditions, the duration varies from 0.1 to $5 \mu \mathrm{s}$, simultaneously the period varies from $1 \mu$ s to $10 \mathrm{~ms}$. The corresponding dynamic spectrogram is shown in Fig. 3(a)the frequencies of the most pronounced discrete lines are $10.8,9.0$, and $8.86 \mathrm{GHz}$. The repetition period of the pulses decreases with increasing microwave power while their amplitude and spectrum are unaffected. Figure 4(b) shows the steady-state regime. The frequency of $\mathrm{cw}$ plasma emission is $8.45 \mathrm{GHz}$ and is independent of the heating power. Figure 4(c) shows another interesting example of the critical behavior not predicted by our theory. Here $B_{\min } / B_{\mathrm{ECR}}$ corresponds to the upper boundary of the cw generation zone, at which the system randomly switches between generation of a quasiperiodic series of pulses and continuous emission.

Although absolute calibration of our detection system is complicated, we may estimate the power of the microwave

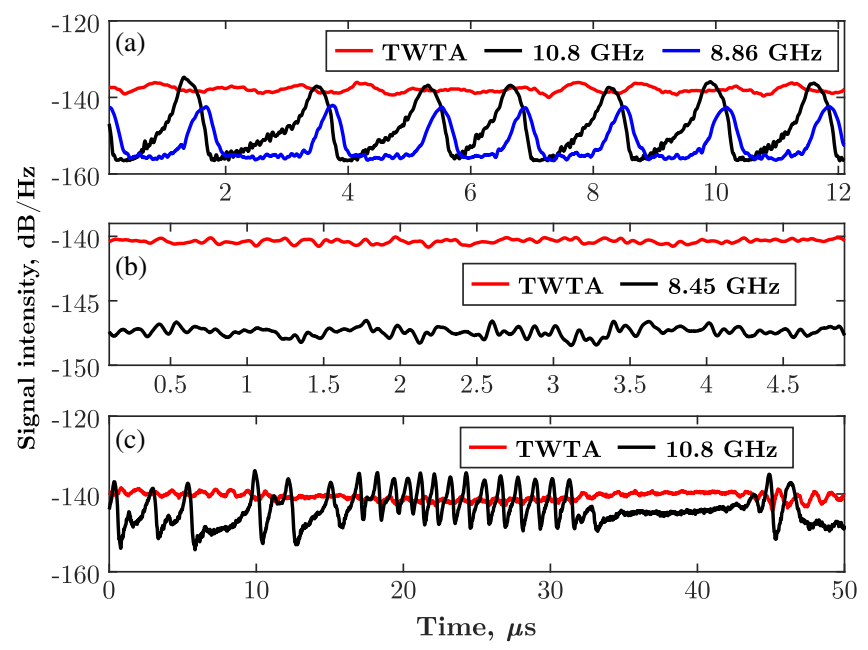

FIG. 4. Intensity of microwave emission in $8-12 \mathrm{GHz}$ band referred to the generation of (a) periodic bursts, (b) cw emission, and (c) spontaneous transition from quasiperiodic bursts to $\mathrm{cW}$ and back. Stray radiation of the heating TWT amplifier at $11.8 \mathrm{GHz}$ is shown with a red line. Three regimes differ only by the value of the magnetic field: $B_{\min } / B_{\mathrm{ECR}}=0.935$ for the burst, 0.947 for the $\mathrm{cw}$, and 0.98 for the transient regimes, correspondingly.

plasma emission in the $\mathrm{cw}$ regime as $\sim 10 \mathrm{~mW}$. This essentially exceeds the power of the spontaneous electron cyclotron emission - as a reference, electrons with energy $200 \mathrm{keV}$ and density $10^{9} \mathrm{~cm}^{-3}$ should emit $\sim 0.3 \mathrm{~mW}$ in our conditions.

Thus, all these data suggest that the observed microwave emission is inherently related to the excitation of electromagnetic waves due to a kinetic cyclotron instability. The most unstable mode is apparently the slow extraordinary wave propagating quasilongitudinally to the external magnetic field and excited in the frequency range between the electron plasma and cyclotron frequencies $[46,47]$. A significant part of the microwave power is measured at frequencies below the cyclotron frequency in the trap center, which indicates that the wave-particle interaction occurs at the relativistically down-shifted cyclotron resonance. To interact with the $\mathrm{cw}$ radiation observed at $8.45 \mathrm{GHz}$, the electrons must have the mean energy of $160 \mathrm{keV}$. For this energy, our theory predicts the Hopf bifurcation at $B_{\min } / B_{\mathrm{ECR}}=\gamma \kappa_{t}^{2}=0.88$. To agree exactly with the experimental value 0.94 , one must assume the electron energy of $200 \mathrm{keV}$.

Examples of the electron precipitation accompanying the microwave emission are shown in Fig. 5 together with the time-averaged energy distributions of axially escaping electrons in different instability regimes. The data demonstrate bursty (a), (b) and continuous (c) precipitation of 4$400 \mathrm{keV}$ electrons including the energies considered above. Moreover, a hump visible at $\sim 200 \mathrm{keV}$ in the stable mode disappears in the burst and $\mathrm{cw}$ regimes. A considerable flux of electrons with energies above $200 \mathrm{keV}$ was previously detected under wider experimental conditions [48]. 

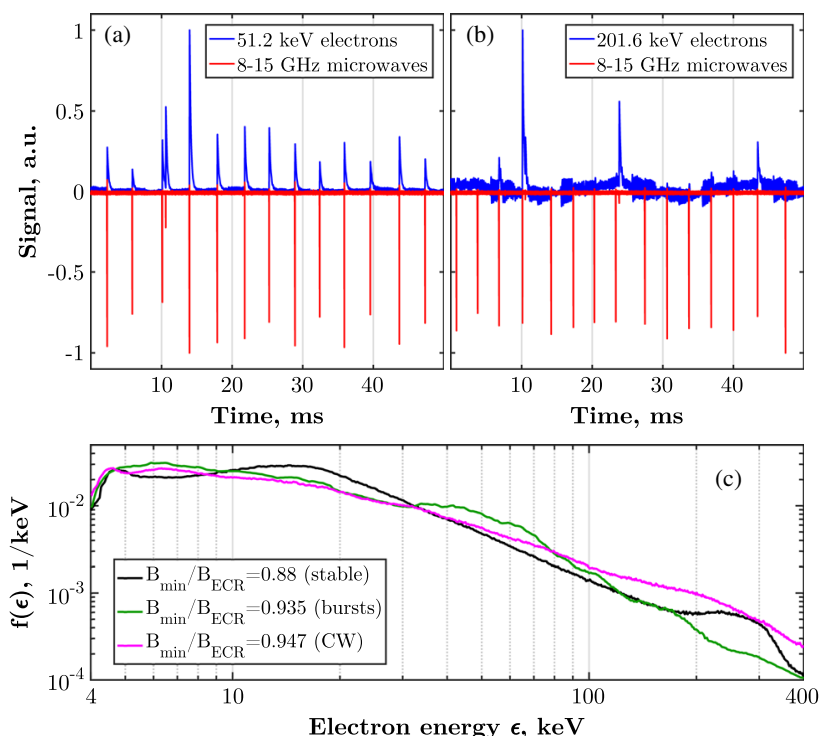

FIG. 5. Waveforms of the electron precipitation through the axial (extraction) mirror with energies of (a) $51.2 \pm 0.25 \mathrm{keV}$ and (b) $201.6 \pm 0.25 \mathrm{keV}$; the microwave emission in the $8-15 \mathrm{GHz}$ range is denoted with red lines. (c) Energy distributions of escaping electrons averaged over $100 \mathrm{~s}$ in the stable, burst, and cw regimes; the distributions are reduced to a unit square by dividing by the total number of detected particles. The measurements are performed using a magnetostatic energy spectrometer with $500 \mathrm{eV}$ resolution [48].

The observed nontrivial dynamics caused by the temporal modulation of the electron distribution function have been previously studied theoretically in the context of space cyclotron masers in planet magnetospheres and other astrophysical objects, and also have much in common with laser excitation mechanisms. Except being of fundamental interest, our results are important for applications such as the development of ECR ion sources. Particle ejections, which are inherent to the burst regime of the cyclotron instability, cause oscillations of the plasma potential and the beam current accompanied with a significant decrease of the average ion charge $[49,50]$. The low-power $\mathrm{cw}$ regime would allow us to avoid these nondesirable effects and improve the ion source performance.

The work was supported by the Academy of Finland under the Finnish Centre of Excellence Programme (Projects No. 213503, No. 296349, No. 311237 for the experiments at the JYFL accelerator laboratory) and by the Russian Science Foundation (Projects No. 16-12-10343 for the data analysis and interpretation, and No. 14-12-01007 for the theoretical modeling). The authors are grateful to Keysight Technologies Inc. for the technical support, and to the referees for their help in improving the Letter.

*ags@appl.sci-nnov.ru

[1] P. A. Bespalov and V. Yu. Trakhtengerts, in Reviews of Plasma Physics, edited by M. A. Leontovich (Consultants
Bureau, New York, 1986), Vol. 10; V. Yu. Trakhtengerts and M. J. Rycroft, Whistler and Alfvén Mode Cyclotron Masers in Space (Cambridge University Press, New York, 2008).

[2] R. A. Treumann, Astron. Astrophys. Rev. 13, 229 (2006).

[3] D. C. Speirs, R. Bingham, R. A. Cairns, I. Vorgul, B. J. Kellett, A. D. R. Phelps, and K. Ronald, Phys. Rev. Lett. 113, 155002 (2014).

[4] D. B. Melrose and G. A. Dulk, Astrophys. J. 259, 844 (1982).

[5] C. S. Wu, Space Sci. Rev. 41, 215 (1985).

[6] L. Vlahos, Sol. Phys. 111, 155 (1987).

[7] P. A. Robinson, Sol. Phys. 134, 299 (1991).

[8] A. J. Willes and P. A. Robinson, Astrophys. J. 467, 465 (1996).

[9] T. S. Bastian, A. O. Benz, and D. E. Gary, Annu. Rev. Astron. Astrophys. 36, 131 (1998).

[10] V. Yu. Trakhtengerts and A. G. Shalashov, Astronomy Reports 43, 540 (1999).

[11] C. Y. Ma, C. Y. Mao, D. Y. Wang, and X. J. Wu, Astrophys. Space Sci. 257, 201 (1998).

[12] R. Bingham, R. A. Cairns, and B. J. Kellett, Astron. Astrophys. 370, 1000 (2001).

[13] M. C. Begelman, R. E. Ergun, and M. J. Rees, Astrophys. J. 625, 51 (2005).

[14] C. Trigilio, P. Leto, G. Umana, C. S. Buemi, and F. Leone, Astrophys. J. Lett. 739, L10 (2011).

[15] W. B. Ard, R. A. Dandl, and R. F. Stetson, Phys. Fluids 9, 1498 (1966).

[16] R. C. Garner, M. E. Mauel, S. A. Hokin, R. S. Post, and D. L. Smatlak, Phys. Rev. Lett. 59, 1821 (1987).

[17] R. C. Garner, M. E. Mauel, S. A. Hokin, R. S. Post, and D. L. Smatlak, Phys. Fluids B 2, 242 (1990).

[18] A. V. Vodopyanov, S. V. Golubev, A. G. Demekhov, V. G. Zorin, D. A. Mansfeld, S. V. Razin, and V. Yu. Trakhtengerts, Plasma Phys. Rep. 31, 927 (2005).

[19] A. V. Vodopyanov, S. V. Golubev, A. G. Demekhov, V. G. Zorin, D. A. Mansfeld, S. V. Razin, and A. G. Shalashov, JETP 104, 296 (2007).

[20] S. V. Golubev and A. G. Shalashov, Phys. Rev. Lett. 99, 205002 (2007).

[21] A. G. Shalashov, S. V. Golubev, E. D. Gospodchikov, D. A. Mansfeld, and M.E. Viktorov, Plasma Phys. Controlled Fusion 54, 085023 (2012).

[22] R. Bingham, D. C. Speirs, B. J. Kellett, I. Vorgul, S. L. McConville, R. A. Cairns, A. W. Cross, A. D. R. Phelps, and K. Ronald, Space Sci. Rev. 178, 695 (2013).

[23] M. E. Viktorov, S. V. Golubev, E. D. Gospodchikov, I. V. Izotov, D. A. Mansfeld, and A. G. Shalashov, Radiophys. Quantum Electron. 56, 216 (2013).

[24] B. Van Compernolle, J. Bortnik, P. Pribyl, W. Gekelman, M. Nakamoto, X. Tao, and R. M. Thorne, Phys. Rev. Lett. 112, 145006 (2014).

[25] B. Van Compernolle, X. An, J. Bortnik, R. M. Thorne, P. Pribyl, and W. Gekelman, Phys. Rev. Lett. 114, 245002 (2015).

[26] M. Viktorov, D. Mansfeld, and S. Golubev, Europhys. Lett. 109, 65002 (2015).

[27] B. Van Compernolle, X. An, J. Bortnik, R. M. Thorne, P. Pribyl, and W. Gekelman, Plasma Phys. Controlled Fusion 59, 014016 (2017). 
[28] A. G. Shalashov, M. E. Viktorov, D. A. Mansfeld, and S. V. Golubev, Phys. Plasmas 24, 032111 (2017).

[29] M. Thumm, Karlsruhe Institute of Technology Report No. KIT-SR 7735, 2016, https://publikationen.bibliothek .kit.edu/1000068193.

[30] P. A. Bespalov and V. Yu. Trakhtengerts, Fizika Plasmy 2, 396 (1976).

[31] H. L. Berk, T. D. Rognlien, and J. J. Stewart, Comments Plasma Phys. Controlled Fusion 3, 95 (1977).

[32] A. V. Gaponov-Grekhov and V. M. Glagolev, V. Yu. Trakhtengerts, JETP 80, 2198 (1981).

[33] P. A. Bespalov, Phys. Scr. T2B, 576 (1982).

[34] Ya. I. Khanin, Fundamentals of Laser Dynamics (Cambridge International Science Publishing Ltd., Cambridge, England, 2006), p. 260.

[35] A. A. Vedenov, E. P. Velikov, and R. Z. Sagdeev, Nucl. Fusion 1, 82 (1961).

[36] W. E. Drummond and D. Pines, Nucl. Fusion Suppl. 3, 1049 (1962).

[37] S. V. Golubev, V.E. Semenov, E. V. Suvorov, and M. D. Tokman, Proceedings of the International Conference on Open Plasma Confinement Systems for Fusion, Novosibirsk, July 14-18, 1993 (World Scientific, Singapore, 1993), p. 261.

[38] D. V. Yakovlev, A. G. Shalashov, E. D. Gospodchikov, A. L. Solomakhin, V. Ya. Savkin, and P. A. Bagryansky, Nucl. Fusion 57, 016033 (2017).

[39] H. Koivisto, P. Heikkinen, V. Hänninen, A. Lassila, H. Leinonen, V. Nieminen, J. Pakarinen, K. Ranttila, J. Ärje, and E. Liukkonen, Nucl. Instrum. Methods Phys. Res., Sect. B 174, 379 (2001).

[40] I. Izotov, D. Mansfeld, V. Skalyga, V. Zorin, T. Grahn, T. Kalvas, H. Koivisto, J. Komppula, P. Peura, and O. Tarvainen, Phys. Plasmas 19, 122501 (2012).
[41] N. K. Bibinov, V. F. Bratsev, D. B. Kokh, V. I. Ochkur, and K. Wiesemann, Plasma Sources Sci. Technol. 14, 109 (2005).

[42] G. Melin, F. Bourg, J. P. Briand, J. Debernardi, M. Delaunay, R. Geller, B. Jacquot, P. Ludwig, T. K. N'Guyen, L. Pin, M. Pontonnier, J. C. Rocco, and F. Zadworny, Rev. Sci. Instrum. 61, 236 (1990).

[43] C. Barué, M. Lamoureux, P. Briand, A. Girard, and G. Melin, J. Appl. Phys. 76, 2662 (1994).

[44] G. Douysset, H. Khodja, A. Girard, and J. P. Briand, Phys. Rev. E 61, 3015 (2000).

[45] I. Izotov, T.Kalvas, H. Koivisto, R. Kronholm, D. Mansfeld, V. Skalyga, and O. Tarvainen, Phys. Plasmas 24, 043515 (2017).

[46] I. Izotov, O. Tarvainen, D. Mansfeld, V. Skalyga, H. Koivisto, T. Kalvas, J. Komppula, R. Kronholm, and J. Laulainen, Plasma Sources Sci. Technol. 24, 045017 (2015).

[47] D. Mansfeld, I. Izotov, V. Skalyga, O. Tarvainen, T. Kalvas, H. Koivisto, J. Komppula, R. Kronholm, and J. Laulainen, Plasma Phys. Controlled Fusion 58, 045019 (2016).

[48] I. Izotov, O. Tarvainen, V. Skalyga, D. Mansfeld, T. Kalvas, H. Koivisto, and R. Kronholm, Plasma Sources Sci. Technol. 27, 025012 (2018).

[49] O. Tarvainen, I. Izotov, D. Mansfeld, V. Skalyga, S. Golubev, T. Kalvas, H. Koivisto, J. Komppula, R. Kronholm, J. Laulainen, and V. Toivanen, Plasma Sources Sci. Technol. 23, 025020 (2014).

[50] O. Tarvainen, T. Kalvas, H. Koivisto, J. Komppula, R. Kronholm, J. Laulainen, I. Izotov, D. Mansfeld, V. Skalyga, V. Toivanen, and G. Machicoane, Rev. Sci. Instrum. 87, 02A703 (2016). 